Si-94-13

\title{
Dynamic Monte Carlo Measurement of Critical Exponents
}

\author{
Z.B. Li円, L. Schülke, and B. Zheng \\ Universität-GH Siegen, D-57068 Siegen, Germany
}

October 27, 1994

\begin{abstract}
Based on the scaling relation for the dynamics at the early time, a new method is proposed to measure both the static and dynamic critical exponents. The method is applied to the two dimensional Ising model. The results are in good agreement with the existing results. Since the measurement is carried out in the initial stage of the relaxation process starting from independent initial configurations, our method is efficient.
\end{abstract}

PACS: 02.70.Lq, 05.70.Jk, 64.60.Ht, 64.60.Fr

\footnotetext{
${ }^{1}$ On leave of absence from Zhongshan University, 510275 Guangzhou, P.R. China
} 
Numerical measurements of critical exponents are usually carried out from samples of configurations in the equilibrium generated with Monte Carlo algorithms. For the static exponents, Binder's method is one of the widely accepted [1], 2]. A traditional way to obtain the dynamic exponent $z$ is to measure the exponential decay of the time correlations for finite systems in the long-time regime [3, 4. At the critical point, except for some special algorithms as e.g. the cluster algorithm [5, 6], one suffers from critical slowing down. Recently, it has been suggested that $z$ may be estimated from the power law decay of the magnetization in a relaxation process on a large enough lattice, in a sufficient large time but before the exponential decay starts $\llbracket 7,8]$.

In the past few years, better understanding has been achieved on the critical relaxation processes even upto the early time of the evolution. A representative example of these processes is that the Ising model initially in random states with a small magnetization is suddenly quenched to the critical temperature and then evolves with the dynamics of model A. It was shown by Janssen et al. [9] with $\varepsilon$-expansion upto two-loop that besides the well-known long-time universal behaviour, there exists another universal stage of the relaxation at earlier time, termed "critical initial slip", which sets in right after the microscopic time scale and eventually crosses over to the long-time regime. For the critical initial slip the characteristic time scale is $t_{0} \sim m_{0}^{-z / x_{0}}$ with $x_{0}$ being a new independent critical exponent and $m_{0}$ the initial magnetization. The scaling behaviour including the increase of the order has been illustrated by a number of authors by Monte Carlo simulationst [11, 10, 12, 13], and also analytical calculations [14, 15, 16, 17].

Of special interest is here the extension of the results in Ref. [9] to finitesize systems 114, 13]. In accordance to the renormalization group analysis for finite-size systems, we expect a scaling relation to hold for the k-th moment of the magnetization in the neibourhood of the critical point [18, 9, 19],

$$
M^{(k)}\left(t, \tau, L, m_{0}\right)=b^{-k \beta / \nu} M^{(k)}\left(b^{-z} t, b^{1 / \nu} \tau, b^{-1} L, b^{-x_{0}} m_{0}\right)
$$

where $t$ is the evolution time, $\tau=\left(T-T_{c}\right) / T_{c}$ is the reduced temperature, $L$ is the lattice size, and $b$ is the spatial rescaling factor. It has been stressed in

${ }^{2}$ After the present letter was completed, we received a preprint of P. Grassberger (Wuppertal, FRG) where the critical initial slip related to damage spreading was investigated and the dynamic exponent $z$ also measured with the Monte Carlo method in both two and three dimensional Ising model. 
Ref. [9] that the initial states must have very short correlation lengths and the initial magnetization $m_{0}$ must be sharply prepared. The scaling relation for the 3-dimensional Ising model has been tested by Monte Carlo simulation [13]. The numerical data fit into the scaling relation nicely, and $x_{0}$ has been determined with satisfactory precision. It was observed that the microscopic time scale is ignorably small, for example, for the heat-bath Monte Carlo algorithm it is smaller than one MC sweep. This clean behaviour of the critical relaxation in its early time indicates a promising new way to measure both the static and dynamic critical exponents, which is similar to Binder's method in equilibrium. In the present letter, we will illustrate this idea for the two dimensional Ising model.

To make the computation simpler and more efficient, we set $m_{0}$ to its fixed point, $m_{0}=0$. Therefore the exponent $x_{0}$ will not enter the calculation. Furthermore, now the time scale $t_{0}=m_{0}^{-z / x_{0}} \rightarrow \infty$, and the critical initial slip gets most prominent in time direction even though the magnetization itself will only fluctuate around zero. The choice of $m_{0}=0$ is essential in the calculation, which allows a more precise and stable measurement of the critical exponents. After generating randomly an initial configuration the system is released to the evolution with the heat-bath algorithm at the critical temperature. We repeat this process with independent initial configurations. The average is taken over the initial configurations with $m_{0}=0$ and zero correlation length.

To determine $z$, we introduce a time-dependent Binder cumulant [2]

$$
U(t, \tau, L) \equiv 1-\frac{M^{(4)}}{3\left(M^{(2)}\right)^{2}} .
$$

Following the scaling relation in Eq.(1), cumulants measured in two different lattices have the simple relation

$$
U\left(t, 0, L_{1}\right)=U\left(b^{-z} t, 0, L_{2}\right)
$$

with $b=L_{1} / L_{2}$. The exponent $z$ can easily be obtained through searching for a time rescaling factor $b^{-z}$ such that the two curves represented by both sides in Eq.(3) collapse. In other words, the cumulant can be described by a scaling function

$$
f\left(t / L^{z}\right)=U(t, 0, L) .
$$


Figure 1: The cumulants $U_{L}$ for $L=8,16$ and 32 (labelled by,$+ \diamond$ and $\star$, respectively) together with the rescaled cumulants of double lattice (solid lines).

In the same way, it is easy to obtain other two scaling functions

$$
\begin{gathered}
g^{(2)}\left(t / L^{z}\right)=L^{2 \beta / \nu} M^{(2)}(t, 0, L), \\
h\left(t / L^{z}\right)=\left.L^{-1 / \nu} \partial_{\tau} \log M^{(2)}(t, \tau, L)\right|_{\tau=0} .
\end{gathered}
$$

With $z$ in hand, $\beta / \nu$ and $\nu$ can be determined by fitting respectively the scaling function $g^{(2)}\left(t / L^{z}\right)$ and $h\left(t / L^{z}\right)$ from different lattices similar as in the determination of $z$. We adopt the derivative of $M^{(2)}(t, \tau, L)$ for the estimation of $\nu$, rather than that of $U(t, \tau, L)$ since the measurement of $M^{(2)}$ is more stable for large $L$. To our knowledge, so far the measurment of the static and dynamic exponents from the dynamic process in the early time has not been investigated. One can easily realize that at time $t \rightarrow \infty$ Binder's way to calculate $\beta / \nu$ and $\nu$ is recovered [3] [1]. Since the measurement can now be carried out already in the initial stage of the relaxation, the method becomes more efficient.

In Fig. 1, we plot the cumulants of the two-dimensional Ising model versus time. The curves corresponding to $L=8,16$ and 32, repectively, are labelled by,$+ \diamond$ and $*$. Each curve is compared with the time-rescaled cumulant of double lattice size given by solid lines. The exponents corresponding to the three pairs of best-fitted curves are $z=2.0969,2.1493$, and 2.1337, respectively. Our best value 2.1337 should be compared with the existing numerical results from [4], $z=2.13(8)$ and from [20], $z=2.14(5)$ and also with that with the $\epsilon$-expansion from [22], $z=2.126$. From the figure one can also see clearly that the scaling relation holds from the very beginning of the time evolution and how remarkably well the method is working.

In Tab. 1, we have summarize the measured exponents $z, 2 \beta / \nu$ and $1 / \nu$ for the 2-dimensional Ising model. They are in good agreement with the exact results [23] and best values for $z$ [4, 22, 20]. To obtain these results, we have averaged over 50000 independent samples in each run and repeated 8 runs to estimate the errors.

\footnotetext{
${ }^{3}$ With a similar procedure used by Binder, the critical point can also be located from the behaviour of $U(t, \tau, L)$ around $\tau=0$.
} 


\begin{tabular}{|c|c|c|c|}
\hline$L_{1} \leftrightarrow L_{2}$ & $z$ & $2 \beta / \nu$ & $1 / \nu$ \\
\hline $8 \leftrightarrow 16$ & $2.0969(20)$ & $0.2480(02)$ & $1.127(08)$ \\
$16 \leftrightarrow 32$ & $2.1493(18)$ & $0.2494(06)$ & $1.058(35)$ \\
$32 \leftrightarrow 64$ & $2.1337(41)$ & $0.2504(29)$ & $0.955(40)$ \\
\hline
\end{tabular}

Table 1: Results for $z, 2 \beta / \nu$ and $1 / \nu$, respectively, from the 2-dimensional Ising model.

Compared with traditional measurements in equilibrium, much less effort is needed with our dynamic Monte Carlo algorithm, since we do not enter the long-time regime where critical slowing down is severe. The efficiency of our method may also be traced back to the simple power law increase of the moments at the early time. For example, it is well known that $M^{(2)}(t, 0, L) \sim t^{(d-2 \beta / \nu) / z}$ when $L$ is large enough [10, 11]. Therefore the scaling relation implies $M^{(2)}(t, 0, L) \sim L^{-d} t^{(d-2 \beta / \nu) / z}$. Similar arguments exists for the cumulant $U(t, \tau, L)$.

In previous works on the critical initial slip [9, 14, 13, 17], the increase of the order and the role of $m_{0}$ in the short- and long-time regimes are intensively discussed. We would like, however, to stress that even in the case of $m_{0}=0$ it is interesting and there exist fruitful applications, as reported in this letter. Our results also provide a further confirmation of the scaling relation discovered by Janssen et al. [9]. Investigations for dynamics in other universality classes should be carried out.

Acknowledgement: The authors would like to thank U. Ritschel for handing useful preprints and helpful discussions in the previous collaboration. Z.B.L. is grateful to the Alexander von Humboldt-Stiftung for a fellowship.

\section{References}

[1] K. Binder and D. W. Heermann, Monte Carlo Simulation in Statistical 
Physics (Springer, 1992).

[2] K. Binder, Z. Phys. B43, 119 (1981).

[3] S. Wansleben and D. P. Landau, Phys. Rev. B 43, 6006 (1991).

[4] J. K. William, J. Phys. A: Math. Gen. 18, 49 (1985).

[5] R. H. Swendsen and J. S. Wang, Phys. Rev. Lett. 58, 86 (1987).

[6] U. Wolff, Phys. Rev. Lett. 62, 361 (1989).

[7] C. Münkel, D. W. Heermann, J. Adler, M. Gofman, and D. Stauffer, Physica A193, 540 (1993).

[8] D. Stauffer, Physica A186, 197 (1992).

[9] H. K. Janssen, B. Schaub, and B. Schmittmann, Z. Phys. B 73, 539 (1989).

[10] D. A. Huse,Phys. Rev. B40, 304 (1989).

[11] K. Humayun and A. J. Bray, J. Phys. A: Math. Gen. 241915 (1991).

[12] N. Menyhárd, J. Phys. A: Math. Gen. 27, 663 (1994).

[13] Z. B. Li, U. Ritschel, and B. Zheng, preprint Si-94-11, to appear in J. Phys. A: Math. Gen..

[14] H. W. Diehl and U. Ritschel, J. Stat. Phys. 73, 1 (1993)

[15] K. Oerding and H. K. Janssen, J. Phys. A: Math. Gen. 26, 3369 (1993); 5295 (1993).

[16] K. Oerding and H. K. Janssen, J. Phys. A: Math. Gen. 27, 715 (1994).

[17] U. Ritschel and H. W. Diehl, Long-time traces of the initial condition in relaxation phenomena near criticality, Essen preprint, 1994.

[18] V. Privmann and M. E. Fisher, Phys. Rev. B30, 322 (1984).

[19] J. Zinn-Justin, Quantum field Theory and Critical Phenomena, (Clarendon Press, 1989).

[20] S. Tang and D. P. Landau, Phys. Rev. B36, 567 (1987).

[21] A. M. Ferrenberg and D. P. Landau, Phys. Rev. B44, 5081 (1991).

[22] R. Bausch, V. Dohm, H. K. Janssen and R. K. P. Zia, Phys. Rev. Lett. 47, 1837 (1981).

[23] R. J. Baxter, Exactly Solved Models in Statistical Mechanics, (Academic Press, 1982). 
This figure "fig1-1.png" is available in "png" format from: http://arxiv.org/ps/hep-th/9410234v1 


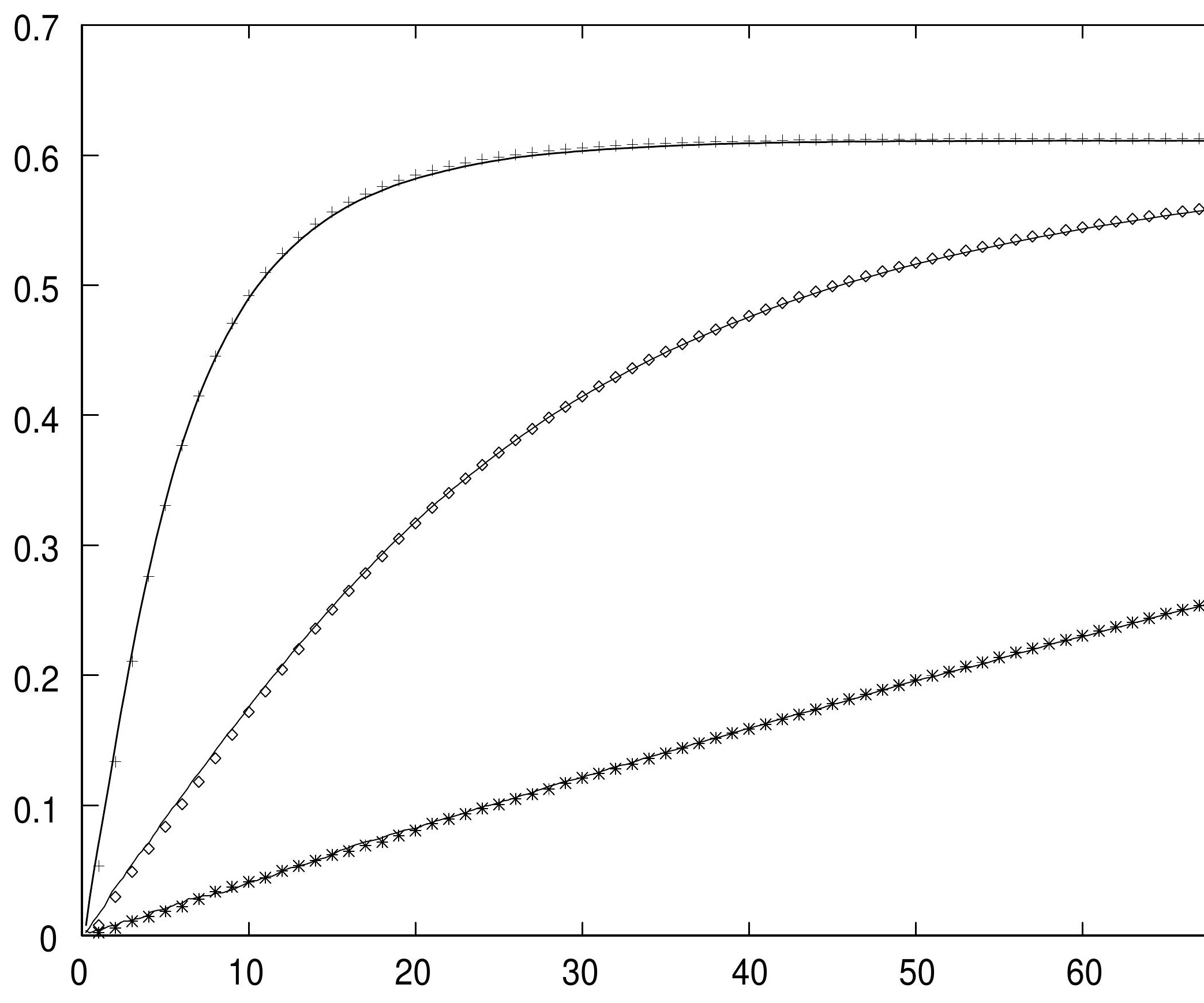

